\title{
Distributed Framework for Tele Health Monitoring System
}

\author{
Bidyut Biman Sarkar \\ Master In Computer Application \\ Techno India Saltlake \\ Kokata, India \\ 9230099359, India \\ bidyutbiman@gmail.com
}

\author{
Sugata Sanyal \\ Tata Institute of Fundamental \\ Research \\ Mumbai, India \\ +912222782591, India \\ sanyal@tifr.res.in
}

\author{
Nabendu Chaki \\ Computer Science \& Engineering \\ University of Calcutta \\ Kolkata, India \\ 9433068073, India \\ nabendu@ieee.org
}

\begin{abstract}
In recent years, it has been a challenge to design and implement remote health care and monitoring services, with an objective to extend instant cost effective treatment and supply of life saving drugs.There are specialized disciplines in medical sciences for consultation, services, and surgery. Tele medicine is one such area of service through which the aged, rural residents and dwellers of remote hilly and highland areas can be benefited. Often, people in these areas suffer from disease like Obstructive Sleep Apnea (OSA), hypertension, cardiovascular problems, obesity, diabetes etc., or respiratory diseases like asthma, pulmonology related to lungs and so on with a high risk of mortality. In this paper, we propose a Petri Net based framework for analysis and integration of tele-health monitoring service systems over wireless and hybrid networks.
\end{abstract}

\section{Categories and Subject Descriptors I.6.5 [Model Development]: Modeling Methodologies}

\section{General Terms}

Management, Design, Experimentation.

\section{Keywords}

Automation; Tele medicine; OSA; sensors; Petri Net; MANET; SOA

\section{INTRODUCTION}

Service to a distressed, an aged, a patient, or a group of persons facing a disaster may possibly require existence of an ad-hoc network with compatible application frame work. The performance of the service granules of the frame work depends on processing overhead at each interface. Service Oriented Architecture (SOA) depends on data and services that are described by metadata, e.g. requirement of an ambulance to relocate a person to a health centre is a discovery of services from a metadata at a reasonable distance, cost and effort without modifying the functional contract of a service. From the software engineering view point coupling among the interfaces should be less and cohesion should be high to form a dynamically executable metadata library. When scope of SOA is distributed,

Permission to make digital or hard copies of all or part of this work for personal or classroom use is granted without fee provided that copies are not made or distributed for profit or commercial advantage and that copies bear this notice and the full citation on the first page. To copy otherwise, or republish, to post on servers or to redistribute to lists, requires prior specific permission and/or a fee.

Conference'11, Month 12, 2011, Kochi, Kerala, India.

Copyright 2011 ACM 978-1-4503-1011-6/11/12...\$10.00. the primary task of the system is to build and maintain a super system metadata of the desired services. For effective communication, the network support should be interoperable, robust, mobile, ad-hoc, fast and cheap [1].

The framework proposed in this paper presents a holistic view of the distributed collaborative tele-health automation process. The projected design attempts to build the basis for offering knowledge-driven quality services at reasonable costs to the end users [2]. The tele-health care repository captures patient data through phone calls, emails, SMS along with their locations, patient-IDs, and disease-code. The collaboration process communicates synchronous messages, audio or videoconference records and sensor transmitted automation transactions. Patients can confer their status with medical experts at their choice and request for additional services. The work-flow system controls and presents the patient data depending on individual patient's data access rights. However, our focus is to design a distributed updatable metadata services for dispersed users over wireless and hybrid networks [3, 4] at a dynamically built low band width communication. e.g., a person in location $\mathrm{x}$ seeks a service of a particular heart specialist must be able to get timely feedback about the availability and appointment. The objective is to configure the metadata repository for efficient use of Mobile Adhoc Network (MANET) at remote sites with the consistent view of repository to all users all the time; it should consume minimum bandwidth for the service with sufficiently high fault tolerant features. The model should support multi platform environments with sufficiently low administrative overhead. To model such stochastic service network we use Petri Net as a tool [5].

In this paper in section 2 we present some recent literature survey on automation of hospital management system, tele-health monitoring system and metadata services. In section 3 the distributed holistic view of the proposed model is presented along with the data services distributed network architecture. A normalized schema of Meta Data is presented in section 4. The coverability analysis of the Meta Data Service model using Petri Net is presented in section 5. The future scope of the study is presented in the concluding section 6 along with the references used in section 6 .

\section{LITERATURE SURVEY}

In the past health service was captive within the four walls of a hospital. In recent times there has been a significant amount of research work on building of scalable metadata storage to extend health services beyond four walls. Some of the technological advances in distributed health care computing are availability of specific sensor devices; wireless based internetworking, broad band services and automation of huge pool of patient data. Despite the success stories of collaborative metadata services to 
our knowledge there is not much social network applications available on medical health services in the literature. We would like to present a distributed updatable frame work for collaborative health care services by deploying a metadata server over ad-hoc and mobile network any where, all the time.

Many approaches like [6], [7], [8], [9] have introduced mobile telemedicine support services. [7], [8] articulate telemedicine system for domestic patient care monitoring through mobile phones. Communication with electro medic devices like patient monitors to transmit vital signals via internet to the hospital for tele-consultation [6]. The system architecture comprises of a medical call centre, a distributed specialists and a hospital linked through a service network [9]. Bandwidth range is vital for all operational purposes to support mobility. Worldwide Interoperability for Microwave Access (WiMAX), ZigBee( IEEE, 802.15.4), wireless personal area network (WPAN), Bluetooth are some of the standards well suited for integration into the medical sensors [10].Sensor networks are used to access patient's state of health under medical observations. Patient Data is continuously transmitted over telecommunication devices like mobile phones, PDAs, PC Systems to doctors, nursing assistants to monitor any abnormal changes of the state of health. Through sensor networks doctors can be quickly tracked in hospitals in case of emergency. Some useful sensors are intra-cranial pressure sensor (ICP), which is primarily used for continuous monitoring of ICP of hydrocephalus patients. It is fully implantable absolute pressure sensor. Glaucoma sensor is a soft contact lens containing a sensor, an antenna and a microprocessor for continuous monitoring and can be performed during activities or patient in sleep etc [11].

Autonomic computing decrease human involvement in computing systems in a similar fashion as its biological counterpart does in a self-managing and self-adaptive systems. Autonomic managers are not only aware of their own managed element but also of their environment, in particular other autonomic elements in the network to improve productivity and reduce costs in hospital operations [12, 13].Target of an autonomic computing is to automate reconfigurable, self-recoverable complex multi-tier distributed systems. The architecture of a complex system composed of a web server, application server and a database server. e.g. a web-server like Apache Tomcat an application server like J2EE and a database server like Oracle or DB2 entails a high level of complexity [14]. Service oriented architecture for Health Research Data Network (HRDN) supports integrated controlled access and privacy protection in a secured end-to-end system for data sharing and analysis. Collection of data and software services are connected through high bandwidth communication infrastructure. Data collections are geographically dispersed and are built on a variety of heterogeneous technologies. Data custodians have ethical and legislative requirements in information integration, while supporting complex requirements arising from use of diverse health data sources [15].

A collaborative metadata sharing on epidemiological and biomedical research is presented in this paper. Comparisons between metadata and data sharing policy, metadata sharing adds additional cost of maintenance and quality improvement. In an ideal situation metadata can be shared in a pre-existing social network with minimal effort and cost with maximum exposure to others. Social networks provide feedback on the collaborators data quality, promptness and availability. The literature presents several examples on social networks to improve the quality of information including success stories [16].

\section{PROPOSED HOLISTIC MODEL VIEW}

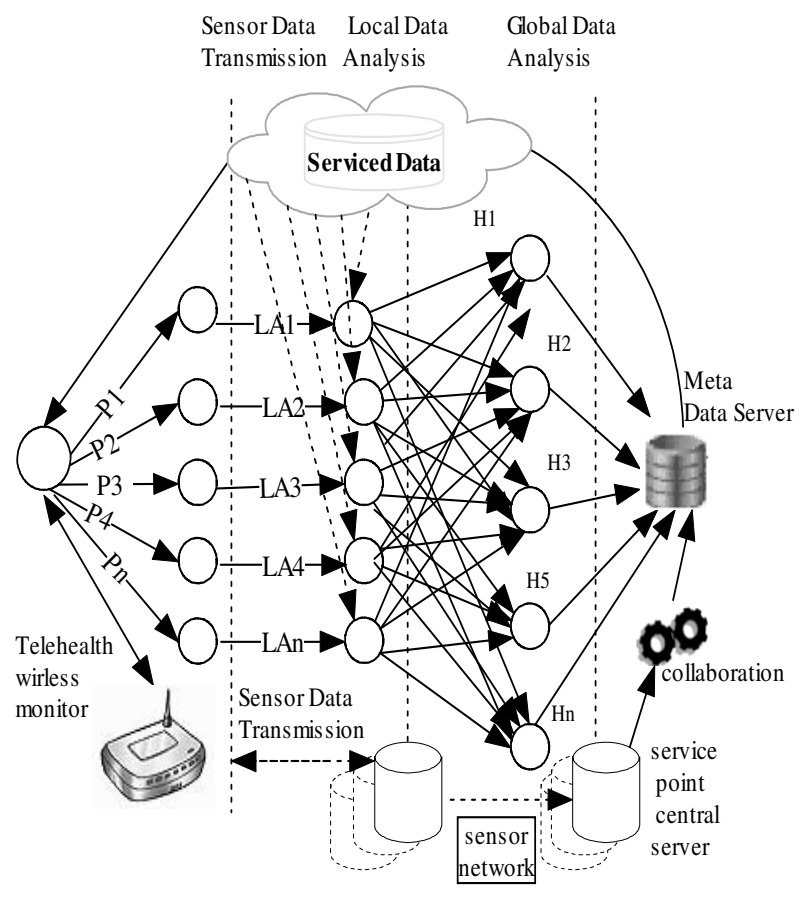

Figure 1. Tele Health Monitoring Distributed MANET

Figure 1 shows the holistic view of the collaborative health monitoring system. Three types of entities are data storage, medical resources and service seekers. Patients $(\mathrm{P} 1, \mathrm{P} 2, \ldots, \mathrm{Pn})$ as data storage resources are attached with sensors like Blood Pressure meter, ECG jacket, etc. and a tele-health wireless monitor within an allowable frequency range are modeled and placed to the repository with the local authority (LA1, LA2,..., LAn), while the medical resources like doctors, nurses, ambulance service etc with mobile phones are modeled as external entities and placed at the repositories with the hospitals $(\mathrm{H} 1, \mathrm{H} 2, \ldots, \mathrm{Hn})$. The mobile service seekers are also considered as external entities and modeled effectively in terms of bandwidth, storage and intermittent connectivity to global data services at service point central server. The metadata server is built in collaboration with LA's, H's and service point central server for future integration purpose. As in any successful data integration process metadata is considered to be critical. Service requests from the registered users in the form of intranet having valid IP address are directed to access the metadata server for a particular service. Depending on the resource requirement the exact repository will be accessed, serviced and the corresponding cost features and service cookies will be preserved for faster and personalized repeat searching and billing purposes.

\subsection{Distributed Network Architecture}

Telemedicine systems in the past are point to point e.g. a rural health centre is mapped to a city hospital and the patient requests are scheduled to doctors belongs to that hospital only. However, with the advent of mobile ad-hoc networks the telemedicine 
systems domain is extended to telemedicine service domain over a distributed network by enabling mobility at the service end like doctor's, remote diagnostic facility, alert services over mobile devices, ambulance services, and soon.

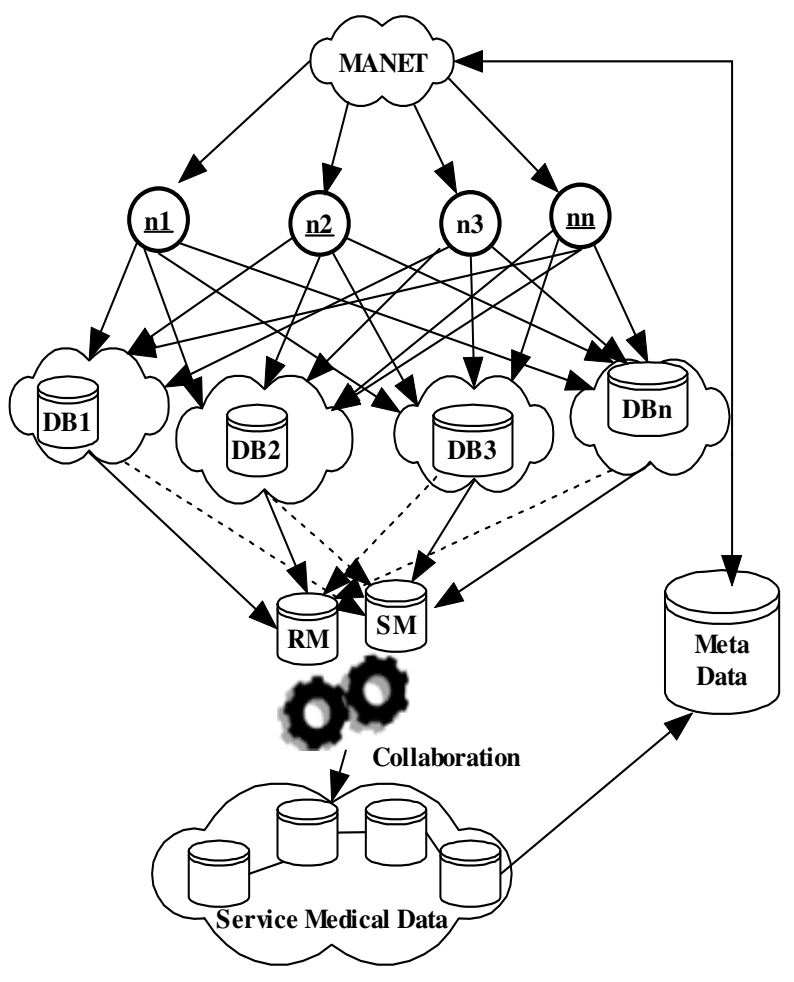

Figure 2. Data services distributed Network

A distributed architecture for data services has access points $(\mathrm{n} 1, \mathrm{n} 2, \ldots, \mathrm{nn})$ and are connected through MANET. It can negotiate multiple service requests over different repositories (DB1,DB2,..,DBn) in a location independent manner through a collaborative and scalable metadata server. The traffic and the connectivity requirement is supported by the MANET and the adhoc network.

In MANET hosts and topology can move frequently, no cellular infrastructure is required, routing takes place through intermediate nodes, mobile nodes dynamically forms multi-hop wireless links without the need of any centralized infrastructure where as $\mathrm{Ad}$ Hoc networks do not require backbone infrastructure support, it is easy to deploy particularly when infrastructure is destroyed [4]. Communication between an access point and a real-time service occurs through TCP/IP connections specified by IP addresses (or host names) and port numbers. For static IP addresses use that number as the host name for accessing the metadata server and the corresponding service medical data. Scalability of each service component maintains its own list of connection links. For all operational purposes the connectivity between the access points and the metadata server can be established through the server manager (SM), data service administrator, Repository Manager (RM) and in case of web based applications the Message client library calls from Web client. The function of RM is to create, upgrade, and check the local and central repositories. The SM handles the transaction management properties over the distributed network like; add, delete or modify of any service request [5]. From the operation management view point RM and
SM should be available throughout the service access points. SM defines the links between service medical data and metadata repositories. In figure 2 , our method suggests multiple medical service data to a single metadata repository. However, for load balancing or for each medical service data to multiple repositories with one default to support individual repositories can be planned. e.g. A simple network management protocol (SNMP) is enabled, which is an application layer protocol standard used to address the schema of the data repository, its objects and its configuration management. SMTP is another application layer protocol that can be used in tandem to send and receive messages through emails for every service function in a secured manner using encrypted channels.

\section{NORMALIZED META DATA SCHEMA}

From the secondary sources it is prevailed that the number of hospitals and Nursing homes with government initiatives and private sector partnership at the state level allover India is around $3300[19,20]$. However, the layered architecture of the health service in India is further extended through primary health centers (PHC) or community health centers $(\mathrm{CHC})$ connected to a district or rural hospitals at layer 1. District Hospitals are connected to State Hospitals at layer 2 and at layer 3 State Level hospitals are connected among themselves. Present strength of PHC's and sub centers is around 25 thousands whereas the number of CHC's is five thousands. Telemedicine programs in India are being actively supported by the Department of Electronics and IT (DIT), Indian Space Research Organization (ISRO), NEC Telemedicine program, Apollo hospitals, Asia Heart Foundation and some more organizations [21].

Considering the volume of common ailment at the eastern part of the Asian Continent in general and India in particular to support the telemedicine program the minimum basic setup at layer 1 PHC should be comprised with the equipments like;

1. Personal Computer and Printer

2. Internet Protocol enabled Video conferencing Kit and clinical devices

3. Telemedicine operating system supported software and a server software

4. Digital ECG device

5. A3 Scanner

6. Digital Microscope and camera

7. Tele-auto scope for remote listening of patient heart beat

8. Gluco meter for blood sugar estimation

9. Haemogram analyzer for blood count and blood film

10. Modem/router/Wifi /Manet etc.

According to WHO the ratio of urban to rural population in India is (28:72). For Successful telemedicine ventures for rural people, a group of urban PHC's should be equipped with a few mobile vans. Our focus is to provide Meta data services to the patients independent of their physical location. In section 3.1 we have described the scope of RM and SM. Now we detail out a primary Meta data schema of the SM and their inter relationship in figure 3. To initiate the process a patient from any location will call or send a SMS to a help line number offered by Government agencies. This needs to be available through all the mobile phone service providers. Patient will pass the information like name, address etc, by calling from a telephone number or health center along with a statement for the type of problem and possible assistance required, etc. 


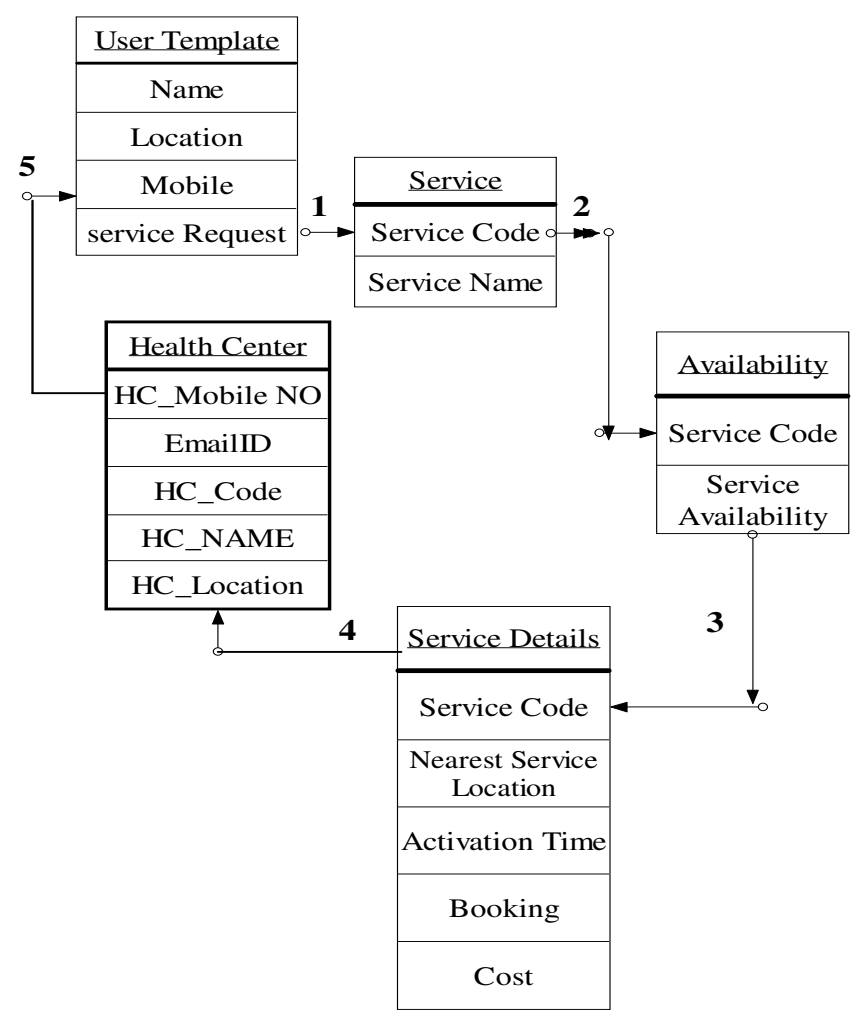

Figure 3. UML Meta Data Activity Diagram

health center reference, type of problem and assistance required. System will generate the template; pass it on to the first level Meta data to service data. Service data checks the availability of the required service. Availability data checks the service details including cost of the service and the information is communicated to the nearest health center as the nodal point. Health Center informs the patient about the service and instructions as to how and from where to start the treatment. E.g. a rural patient suffering from a chest pain calls for a service using the mobile phone to the $\mathrm{HC}$ with the help of $(1,2)$ of figure 3 . Severity demands mobile vans belong to the HC. After initial diagnosis with the telemedicine unit at Van/HC blood pressure 98/59, heart rate 72 , and blood glucose $147 \mathrm{mg} / \mathrm{dL}$ recorded and sent to (3) of figure 3. Remote cardiologist of the meta data source checks the patient records and medication dosage instructed to the HC registered doctors along with the cost of service through (4) of figure 3 . In turn health centre inform the patient party.

A model is a representation of a specific purpose that has an abstraction, a simplification and that represents relationships amongst the components of the system being modeled. Due to complex distributed nature of the health monitoring system, we propose to model the system to understand how the activities are executed now and in future. Good models depend on data quality, tools and clear thinking with multiple medium of operations like mobile phones, remote diagnostic devices, mobile vans with VSAT connectivity, remote medical practitioners and so on. The logical model helps in understanding the process and also lays strong foundation for the physical structures. Petri Net is often used as a meta modeling tool to study and analyze the complexity among the large number of collaborative devices and processes. Petri Net supports a unique combination of graphical as well as mathematical formalism to model a dynamic system. Rich mathematical foundations permit in-depth analysis of work-flow nets.

\section{MODEL FOR THE DATA SERVICE}

Petri Net is a meta modeling tool to model discrete events. The model describes the states, events, conditions, choice, iterations and parallelism. The two types of nodes are called places and transitions. Places and transitions are connected via arcs. Places are represented by circles, transitions by bars..

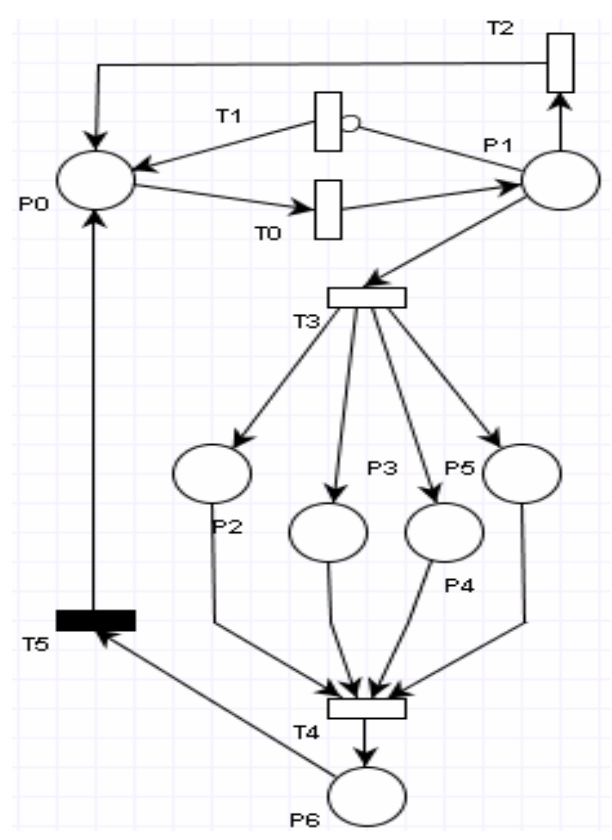

Figure 4. PN model of the distributed Data Services Network

The formal definition of a Petri Net (PN)is described by the 4tuple presented as $\mathrm{PN}=(\mathrm{P}, \mathrm{T}, \mathrm{D}-, \mathrm{D}+)$, where, $\mathrm{P}$ is the set of places and $|\mathrm{P}|=\mathrm{m}, \mathrm{T}$ is the set of. $|\mathrm{T}|=\mathrm{n}, \mathrm{D}-=\mathrm{P} \times \mathrm{T}, \mathrm{N}$ is the pre incidence matrix that specifies the arcs directed from places to transitions. $\mathrm{D}+=\mathrm{T} \times \mathrm{P}, \mathrm{N}$ is the post incidence matrix that specifies the arcs directed from transitions to places [18]. Entities in the modeling process are patients, medical records and mobile service seekers. The stochastic model of the PN is designed with the patients, medical records and service seekers with seven places and six transitions to check the boundedness, liveness, safeness, and to perform the reachability analysis of the Net.

Table 1. Place \& Transition Function of the model

\begin{tabular}{|c|l|c|l|}
\hline \multicolumn{2}{|c|}{ Place } & \multicolumn{2}{c|}{ Transition } \\
\hline P0 & $\begin{array}{l}\text { Service seeking } \\
\text { external node }\end{array}$ & T0 & $\begin{array}{l}\text { Transition for metadata } \\
\text { search }\end{array}$ \\
\hline P1 & Metadata Server & T1 & $\begin{array}{l}\text { Timed Transition } \\
\text { service request }\end{array}$ \\
\hline P2 & $\begin{array}{l}\text { Medical service data } \\
\text { repository }\end{array}$ & T2 & $\begin{array}{l}\text { Timed Transition for no } \\
\text { service support }\end{array}$ \\
\hline P3 & $\begin{array}{l}\text { Medical service data } \\
\text { repository }\end{array}$ & T3 & $\begin{array}{l}\text { Timed Transition for } \\
\text { service support }\end{array}$ \\
\hline P4 & $\begin{array}{l}\text { Medical service data } \\
\text { repository }\end{array}$ & T4 & $\begin{array}{l}\text { Timed Transition for } \\
\text { service attainment }\end{array}$ \\
\hline P5 & $\begin{array}{l}\text { Medical service data } \\
\text { repository }\end{array}$ & T5 & $\begin{array}{l}\text { Immediate service } \\
\text { attainment }\end{array}$ \\
\hline P6 & Availability of service & & \multicolumn{2}{|l}{} \\
\hline
\end{tabular}


Initially, there would be no token at any of the 6 places. On call from a patient the transition $\mathrm{T} 1$ generates a token through the inhibitor are. Patient monitoring is abstracted from the modeling process. Let us now list the transitions and place in table 1.

\subsection{Incidence Matrices of the Petri Net}

Table 2 (b). Post incidence Matrix $\left[\mathrm{D}^{+}\right]$

\begin{tabular}{|c|c|c|c|c|c|c|}
\hline & T0 & T1 & T2 & T3 & T4 & T5 \\
\hline P0 & 1 & 0 & 0 & 0 & 0 & 0 \\
\hline P1 & 0 & 0 & 1 & 1 & 0 & 0 \\
\hline P2 & 0 & 0 & 0 & 0 & 1 & 0 \\
\hline P3 & 0 & 0 & 0 & 0 & 1 & 0 \\
\hline P4 & 0 & 0 & 0 & 0 & 1 & 0 \\
\hline P5 & 0 & 0 & 0 & 0 & 1 & 0 \\
\hline P6 & 0 & 0 & 0 & 0 & 0 & 1 \\
\hline
\end{tabular}

pipe2 [17]. Let us now study some of the properties and behavior of the model based on the Coverage Graph presented in Figure 4. Reachability graph is declared safe, if the number of tokens at that place is either 0 or 1 . In our case the graph clearly shows that any of the places [P0 - P6] has a maximum token count 1 or 0 and is declared safe and as all the places in the net are safe, the net as a
Table 2 (a). Pre incidence Matrix [D”]

\begin{tabular}{|c|c|c|c|c|c|c|}
\hline & T0 & T1 & T2 & T3 & T4 & T5 \\
\hline P0 & 0 & 1 & 1 & 0 & 0 & 1 \\
\hline P1 & 1 & 0 & 0 & 0 & 0 & 0 \\
\hline P2 & 0 & 0 & 0 & 1 & 0 & 0 \\
\hline P3 & 0 & 0 & 0 & 1 & 0 & 0 \\
\hline P4 & 0 & 0 & 0 & 1 & 0 & 0 \\
\hline P5 & 0 & 0 & 0 & 1 & 0 & 0 \\
\hline P6 & 0 & 0 & 0 & 0 & 1 & 0 \\
\hline
\end{tabular}

Table 2 (c). Resultant Matrix $\left[\mathrm{D}=\mathrm{D}^{-}-\mathrm{D}^{+}\right]$

\begin{tabular}{|c|c|c|c|c|c|c|}
\hline & T0 & T1 & T2 & T3 & T4 & T5 \\
\hline P0 & -1 & 1 & 1 & 0 & 0 & 1 \\
\hline P1 & 1 & 0 & -1 & -1 & 0 & 0 \\
\hline P2 & 0 & 0 & 0 & 1 & -1 & 0 \\
\hline P3 & 0 & 0 & 0 & 0 & -1 & 0 \\
\hline P4 & 0 & 0 & 0 & 1 & -1 & 0 \\
\hline P5 & 0 & 0 & 0 & 1 & -1 & 0 \\
\hline P6 & 0 & 0 & 0 & 0 & 1 & -1 \\
\hline
\end{tabular}

The places P0 and P1 are indicating the patient and meta data server respectively. The places P2 through P5 indicate five numbers of medical service data repositories. P6 having tokens indicate the availability of requested service. The transitions T3 and $\mathrm{T} 4$ are stochastic in nature as the requested service may not be available in any of the RM or SM indicated in section 3.1.

Table 2(a) is the pre incidence matrix indicating the state transitions. Table 2(b) represents the post incidence matrix and Table 2(c) represents the combined matrix. The resultant matrix illustrates that there is 1 in row P6 and column T4. This indicates that the service is available. When the service is availed, transition $\mathrm{T} 5$ fires and a token is passed from $\mathrm{P} 6$ to $\mathrm{P} 0$.

\subsection{Coverage Graph of the Petri Net Model}

Table 3 stores the markings corresponds to the places P0, P1, P2, P3, P4, P5 and P6. The tabulation to view state markings along with state transition edges are generated by the Petri Net tool

Table 3: State Marking for the coverage Graph

\begin{tabular}{|c|c|c|c|}
\hline State & Marking & Edges From & Edges To \\
\hline S0 & 1000000 & S1(T2), S5(T5) & S1(T0), S2(T1) \\
\hline S1 & 0000001 & S0(T0) & S3(T3), S0(T2) \\
\hline S2 & $\omega 000000$ & S0(T1), S4(T2) & S4(T0), S2(T1) \\
\hline S3 & 0111100 & S1(T3) & S5(T4), S6(T1) \\
\hline S4 & $\omega 000001$ & S2(T0) & S7(T3),S8(T0), S2(T2) \\
\hline S5 & 0000001 & S3(T4) & S0(T5) \\
\hline S6 & $\omega 111100$ & S3(T1) & S9(T0), S10(T4),S7(T1) \\
\hline S7 & $\omega \omega \omega \omega 00$ & S4(T3),S6(T1), & S9(T10), S11(T4),S7(T1) \\
\hline S8 & $\omega 00000 \omega$ & S4(T3,T12) & S12(T3),S8(T0,T1,T2) \\
\hline S9 & $\omega \omega \omega \omega \omega 01$ & S6(T0),S7(T0) & S7(T3),S12(T0), \\
\hline S10 & $\omega 1111 \omega 0$ & S6(T4) & S10(T4),S7(T2) \\
\hline S11 & $\omega \omega \omega \omega \omega \omega 0$ & S7(T4) & S11(T5) \\
\hline S12 & $\omega \omega \omega \omega \omega 0 \omega$ & S8(T3),S9(T10) & S4(T4), \\
\hline S13 & $\omega \omega \omega \omega \omega \omega 1$ & S9(T4) & S12(T0,T1,T2,T3) \\
\hline S14 & $\omega \omega \omega \omega \omega \omega \omega$ & S12(T4) & S13(T5) \\
\hline
\end{tabular}

whole can be declared safe. Boundedness is a generalized property of safeness. The limitation of token numbers in a place is restricted to 1 and hence it is bounded. Conservation property of a Petri Net model checks that the number of tokens remains constant before and after the execution. In our case it is 1 . The liveness property of a Petri Net will check the possibility of deadlock states. For our case the possibility of deadlock or live lock cannot be ruled out. If T4 is indecisive over a long time, livelock may take place and this is to be checked.

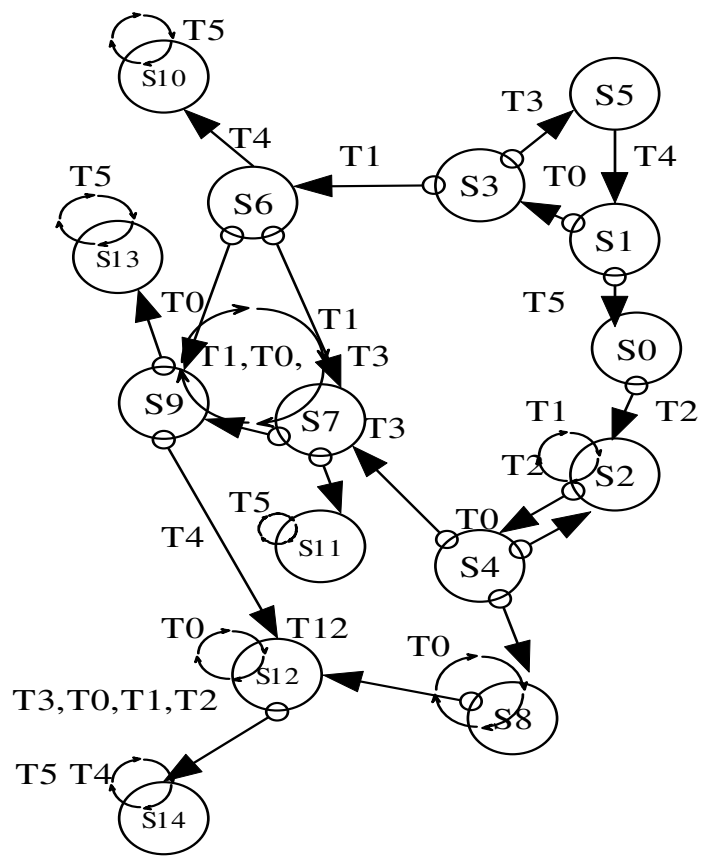

Figure 5. Coverage graph of the Services Network model

\section{CONCLUSION}

This paper aims to address a small step for Information Technology but it is a giant leap for healthcare. The challenges of convergence in health service to a predictable point at a reasonable cost and time. Patients with chronic syndrome will 
improve symptom management, avenue to assess and compliance to adhere to prescribed regimens of care. The proposed Petri Net based model helps towards analysis, collaboration and integration of remote health monitoring services over wireless and hybrid network to achieve the target of converged health services. The high speed networks offer the basic infrastructure for the collection, organization, storage, analysis, and distribution of dynamic operational data. The proposed collaborative approach would add a new dimension to the health services. In order to illustrate this emerging paradigm, several different applications over various service domains have been reviewed. The current work lays the foundation towards building a framework for efficient management of dynamic distributed health service metadata. A national level metadata health grid can also be formed and shared by health care providers and beneficiaries for easy access of the meta data services.

\section{REFERENCES}

[1] Deepti Parachuri, Majumdar Bijoy, Mysore Ujval, 2007. Semantic SOA in Action: Services Computing. In Proceedings of the IEEE International Conference, SCC2007. ISBN: 0-7695-2925-9.

[2] Benjamin F. Miller, Psy. D, 2009. Collaborative Care Research Network. In Proceedings of the Research Development Conference, (Publication No. 11-0067, July 2011).

[3] Alessandro Bianchi, Sebastiano Pizzutilo, 2010. Studying MANET through a Petri Net-based Model. In Proceedings of the 2nd International Conference on Evolving Internet, 220225, ISBN: 978-0-7695-4185-3

[4] Zhang Li, Zhang Yang, 2010. A Wireless Ad Hoc Overlay Networks Indexing Mechanism Based on Petri Nets theory. In Proceedings of the 2nd Second International Conference on evolving Internet, 220-225, ISBN: 978-07695-4185-3.

[5] Bidyut Biman Sarkar, Nabendu Chaki, 2010. Transaction Management for Distributed Database Using Petri Nets. In International Journal of Computer Information Systems and Industrial Management Applications (IJCISIM), Vol.2, 6976, ISSN: 2150-7988.

[6] Xiao Y, et al., 2000. Design and Evaluation of a real-time Mobile Telemedicine System for Ambulance Transport. In Journal of High Speed Networks, vol. 9, no. 1, 47- 56, ISSN:0926-6801 (Print), 1875-8940 (Online)

M. V. M. Figueredo, J. S.Dias, 2004. Mobile Telemedicine System for Home Care and Patient Monitoring. In Proc. of the 26th International Conference of the IEEE EMBS, vol.2, 3387-3390, ISBN:3642022669.
[7] Jocelyn Adams, et al., 2007, Consideration of Security in Tele health Wireless Sensor Network Monitoring Systems. In Proceeding of the third International Conference on Telehealth IASTED, ACM digital library, ISBN: 978-0-88986-668-3.

[8] Tepei Tang, Jeng Ku, 2007, Mobile Care: Telemedicine Based On Medical Grid and Mobile Network. In Proc. of International Conference on Wireless Communications, Networking and Mobile Computing, 3160-3163, ISBN: 9781-4244-1311-9.

[9] Evans Pughe C, 2003, ZigBee wireless standard. In IEEE Review, vol.49, no.3, 28-31, ISSN:0953-5683.

[10] Chee-yee Chong, Srikanta P.Kumar, 2003, Sensor Networks Evolution, Opportunities, and Challenges. In Proc. of the IEEE Transactions, 91(8), ISSN:1247-1256

[11] Kephart J. O. and Chess D. M, 2004, The vision of Autonomic computing. In GCC workshop, Springer(Berlin, Heidelberg, NW), 41-50, ISSN:0302-9743

[12] Rajiv Khosla, Belal Chowdhury, 2004, Real-time RFID based intelligent healthcare diagnosis system. In International conference on Medical Biometrics, SpringerVerlag, 1189-1195, ISSN(online):11894-0814

[13] Bigus, J. P., Schlosnagle, et al.,2002, A toolkit for building Multi-agent autonomic systems. In IBM System Journal, 41(3), 350-371, DOI: $10.1147 / \mathrm{sj} .413 .0350$

[14] Kerry Taylor, Christine M. O. Keefe, John Colton, 2006, A Data Network for Health e-Research. In Proceedings of the ECEH of European Research Net on eHealth, Vol. P-91, 215-226, ISBN 978-3-88579-185-0

[15] Nabendu Chaki, Bidyut Biman Sarkar, 2010, Virtual Data Warehouse Modeling Using Petri Nets for Distributed Decision Making. In Journal of Convergence Information Technology (JCIT), vol.5(5), 8-21, ISSN: 1975- 9320

[16] P. Bonet, C.M. Llado, et al., 2007, PIPE-v2.5: A Petri Net Tool for Performance Modeling. In Proceedings of the XXXIii Conference Latinoaméricana de Informática, ISBN: 978-9968-9678-9-1

[17] W.M.P Vander Aalst, 1994, Putting high-level Petri Nets to Modeling CLEI, Work in Industry. In Journal of Elsevier Computers in Industry, vol.25, 45-54, ISBN:9789968967891

[18] List of Hospitals in India. www.hindustanlink.com/hospitalsindia/index.htm

[19] Health Statistics in India, 2005. IN Central Bureau of Health Intelligence, HEALTH INFORMATION OF INDIA, http://cbhidghs.nic.in/content.asp

[20] Country Health System Profile: India, 2009, In World Health Organization report SEARO, http://www.searo.who.int /EN/Section313/ Section 1519.htm 